\title{
The Accurate Prediction of Protein Family from Amino Acid Sequence by Measuring Features of Sequence Fragments
}

\author{
HUIXIAO HONG, QILONG HONG, ${ }^{2}$ ROGER PERKINS, ${ }^{3}$ LEMING SHI, HONG FANG, ${ }^{3}$ \\ ZHENQIANG SU, YVONNE DRAGAN, JAMES C. FUSCOE, and WEIDA TONG ${ }^{1}$
}

\begin{abstract}
The rapid advances in proteomic analyses coupled with the completion of multiple genomes have led to an increased demand for determining protein functions. The first step is classification or prediction into families. A method was developed for the prediction of protein family based only on protein sequence using support vector machine (SVM) models. In these models, the amino acids were classified into three categories (apolar, polar, and charged). Consecutive fragments ranging from one to five were annotated by amino acid type to define the protein features of each protein. SVM models were constructed based on the protein features of a training set of proteins and then examined with an independent set of proteins. The approach was tested for 20 protein families from the iProClass database of Protein Information Resources (PIR). For two-class SVM models, an average prediction accuracy of 0.9985 was achieved, while for multi-class SVM models an accuracy of 0.9941 was achieved. This study demonstrates that SVM based methods can accurately recognize and predict the protein family to which a sequence belongs based solely on its primary amino acid sequence.
\end{abstract}

Key words: prediction, protein family, protein feature, recognition, sequence.

\section{INTRODUCTION}

$\mathbf{T}$ HERE IS A CRITICAL NEED for reliable and efficient methods of protein family prediction and classification from protein sequences in response to the overwhelming volume of sequence data generated from genomic and proteomic research. Although this sequence data is available in databases such as Protein Information Resources (PIR), knowledge about the function of these proteins is incomplete and computational methods are needed to aid in protein characterization. One promising strategy for protein classification

\footnotetext{
${ }^{1}$ Division of Systems Toxicology, National Center for Toxicological Research, U.S. Food and Drug Administration, Jefferson, Arkansas.

${ }^{2}$ Department of Mechanical Engineering, University of Arkansas, Fayetteville, Arkansas.

${ }^{3}$ Division of Bioinformatics, ICF International at National Center for Toxicological Research, U.S. Food and Drug Administration, Jefferson, Arkansas.

The views presented in this article do not necessarily reflect those of the U.S. Food and Drug Administration.
} 
involves the classification of uncharacterized proteins into existing protein families that share common structural and functional features.

Most of the published methods are based on computationally expensive multiple sequence alignment. The most popular approach (Sgourakis et al., 2005; Karchin et al., 2002; Baldi et al., 1994; Krogh et al., 1994) is based on Hidden Markov Model (HMM). Although HMM provides a solid statistical foundation for building classification models using multiple sequence alignment, it is very expensive in terms of computational time. This leads to a low efficiency for protein family prediction, since each of the sequences must be aligned with all the sequences in the training set prior to the prediction. Furthermore, prediction accuracy must by necessity be very high, because the large number of proteins implies that otherwise a high false misclassification rate would occur. For example, in a model with an accuracy of 0.95 , prediction of 10,000 proteins may result in 500 misclassifications. Alignment-independent methods have been developed for the classification of proteins, but the prediction accuracy of current models has not surpassed 0.92 (Vries et al., 2004; Lapinsh et al., 2002). Based on the limitations inherent in the current classification methods, a set of protein features, defined as the frequency of one to five consecutive amino acids in a protein sequence compared with random sequences, were assessed for accurate protein classification and prediction. These protein features were then used to construct support vector machine (SVM) models for protein family prediction and classification. Twenty protein families in the iProClass database (Wu et al., 2004a) of PIR were used to test the approach.

\section{METHODS}

\subsection{Calculation of protein features}

The sequence of amino acids of a protein implicitly encodes information that determines its threedimensional structure, which explicitly determines its biological function. Protein folding is largely determined by the inter-atomic interactions between adjacent residues (Anfinsen, 1973). For example, Valine, Isoleucine, and Threonine are beta-branched amino acids that are responsible for making beta-sheet turns in a protein structure. The 20 amino acids are classified into three categories based on commonality of chemical properties that determine their residue-to-residue interactions. The eight amino acids Ala, Val, Leu, Ile, Pro, Phe, Trp, and Met are relatively apolar residues that interact with other residues, mainly by Van der Waals interaction. A second category of amino acids include those with charged residues, Asp, Glu, Lys, Arg, and His, where hydrogen bonding, charge-dipole, and dipole-dipole interactions will predominate. Finally, Gly, Ser, Thr, Cys, Tyr, Asn, and Gln comprise a third category of uncharged residues that will tend to similarly contribute to stable folding primarily through dipole-dipole and hydrogen bonding interactions. A protein sequence, $\mathrm{S}$, of length $n$ amino acids, is broken into fragments of length one to five consecutive amino acids. The frequencies of the fragments are used to define the features of a protein as the relative abundance of each fragment, calculated as:

$$
f_{L}^{i}=\frac{P_{L}}{T_{L}} N_{L}^{i}
$$

where $\mathrm{L}$ is the length of a fragment, $1-5$ here; $\mathrm{i}$ indicates the fragment $\mathrm{i}$ with length $\mathrm{L} . N_{L}^{i}$ is the number of fragment $i$ found in $\mathrm{S} ; T_{L}$ is the total number of fragments with length $\mathrm{L}$ found in $\mathrm{S} ; P_{L}$ is the number of possible fragments with length $\mathrm{L}$. When $\mathrm{L}=1$ or 2 , amino acids are used. When $\mathrm{L}=3$ or 4 or 5 , the three types of amino acids are used to reduce the number of possible fragments. For example, if a protein sequence, S, has 2001 amino acids, there are a total of 2000 fragments of length two consecutive amino acids in $\mathrm{S}\left(\mathrm{T}_{L}=2000, \mathrm{~L}=2\right)$. The possible number of fragments of two consecutive amino acids $(\mathrm{L}=2)$ is 400 because there are 20 different amino acids $\left(\mathrm{P}_{\mathrm{L}}=400\right)$. Therefore, if 20 fragments of consecutive dipeptides Ala-Leu (Alanine-Leucine) are found in the sequence $\mathrm{S}$, the frequency of dipeptide Ala-Leu in the sequence $S$ is calculated as $f=400 * 20 / 2000=4.771$ features listed in Table 1 were calculated and used in this article. Feature value of one indicates that the abundance of the fragment in a sequence is the same as in random sequences, and this assumes a uniform background feature distribution. Generally, a higher feature value implies greater fragment abundance in a sequence compared to random sequences.

The 771 protein features used in this study were calculated for every protein in the 20 families using a MatLab code. The MatLab code accepts a file in FASTA format and outputs a text file for model construction and prediction, in which each row is for a protein and each column is for one of the 771 protein features. The MatLab code is available upon request. 
Table 1. Protein Features Used in this Article

\begin{tabular}{|c|c|c|}
\hline Type & Calculation of features & Number of features \\
\hline A & $\begin{array}{l}\text { Number of individual amino acids in a protein }(\mathrm{A}=\text { any } \\
\text { of the } 20 \text { amino acids })\end{array}$ & 20 \\
\hline $\mathrm{AB}$ & $\begin{array}{l}\text { Number of individual available connections of two amino } \\
\text { acids (A, B = any of the } 20 \text { amino acids) }\end{array}$ & 400 \\
\hline $\mathrm{ABC}$ & $\begin{array}{l}\text { Number of individual available connections of three classes } \\
\text { of amino acids (A, B, C = any of the } 3 \text { classes of amino acids) }\end{array}$ & 27 \\
\hline $\mathrm{ABCD}$ & $\begin{array}{l}\text { Number of individual available connections of four classes } \\
\text { of amino acids (A, B, C, D = any of the } 3 \text { classes of amino acids) }\end{array}$ & 81 \\
\hline $\mathrm{ABCDE}$ & $\begin{array}{l}\text { Number of individual available connections of five classes } \\
\text { of amino acids (A, B, C, D, E = any of the } 3 \text { classes of amino acids) }\end{array}$ & 243 \\
\hline
\end{tabular}

\subsection{Sequences of the 20 protein families}

The 4383 protein sequences of 20 families used in this paper were downloaded from iProClass database of PIR (http://pir.georgetown.edu/) in the FASTA format. Information regarding the 20 protein families is listed in Table 2. All sequences in each family in the iProClass database were downloaded. It was noted that the number of sequences in iProClass database is smaller than the number of sequences in PIRSF database (Wu et al., 2004b) for some protein families. The iProClass database was used, because it provides a function to easily download multiple sequences. The protein sequences in each family were put into one file. The redundant protein sequences were removed from the file prior to protein feature generation. The 20 files of unique protein sequences in FASTA format were then passed to the MatLab code to generate profiles of the 771 protein features for each protein family. Profiles of protein features in each of the 20 families were randomly divided into two portions evenly to serve as the training and the testing samples respectively (for odd number of proteins, the number of proteins in the training part is one more than that

Table 2. Details on Training and Testing of Protein Sequences of 20 Protein Families

\begin{tabular}{|c|c|c|c|c|c|c|c|}
\hline \multirow[b]{2}{*}{$I D$} & \multirow[b]{2}{*}{ Group } & \multirow[b]{2}{*}{ Family } & \multirow[b]{2}{*}{ Sequences } & \multirow[b]{2}{*}{ Redundant sequences } & \multicolumn{3}{|c|}{ Unique sequences } \\
\hline & & & & & All & Training & Testing \\
\hline 1 & 1 & Pirsf000048 & 139 & 12 & 127 & 64 & 63 \\
\hline 2 & 1 & Pirsf000071 & 155 & 19 & 136 & 68 & 68 \\
\hline 3 & 1 & Pirsf000078 & 384 & 65 & 319 & 160 & 159 \\
\hline 4 & 1 & Pirsf000090 & 316 & 18 & 298 & 149 & 149 \\
\hline 5 & 1 & Pirsf000099 & 283 & 8 & 275 & 138 & 137 \\
\hline 6 & 1 & Pirsf000363 & 246 & 9 & 237 & 119 & 118 \\
\hline 7 & 1 & Pirsf000806 & 94 & 1 & 93 & 47 & 46 \\
\hline 8 & 1 & Pirsf001336 & 133 & 7 & 126 & 63 & 63 \\
\hline 9 & 1 & Pirsf001401 & 357 & 29 & 328 & 164 & 164 \\
\hline 10 & 1 & Pirsf001423 & 143 & 7 & 136 & 68 & 68 \\
\hline 11 & 2 & Pirsf000050 & 213 & 6 & 207 & 104 & 103 \\
\hline 12 & 2 & Pirsf000055 & 156 & 1 & 155 & 78 & 77 \\
\hline 13 & 2 & Pirsf000059 & 501 & 17 & 484 & 242 & 242 \\
\hline 14 & 2 & Pirsf000076 & 96 & 2 & 94 & 47 & 47 \\
\hline 15 & 2 & Pirsf000098 & 187 & 14 & 173 & 87 & 86 \\
\hline 16 & 2 & Pirsf000379 & 140 & 27 & 113 & 57 & 56 \\
\hline 17 & 2 & Pirsf000443 & 205 & 20 & 185 & 93 & 92 \\
\hline 18 & 2 & Pirsf001124 & 151 & 5 & 146 & 73 & 73 \\
\hline 19 & 2 & Pirsf001237 & 127 & 5 & 122 & 61 & 61 \\
\hline 20 & 2 & Pirsf002451 & 357 & 65 & 292 & 146 & 146 \\
\hline Total & & 20 families & 4383 & 337 & 4046 & 2028 & 2018 \\
\hline
\end{tabular}


in the testing part). The training and the testing of the 20 protein families used in this article are listed in Table 2.

\subsection{Support vector machine models}

SVM is a popular technique for classification (Cortes Vapnik, 1995; Keerthi and Lin, 2003). It embodies the structural risk minimization principle, which has been shown to be superior to traditional empirical risk minimization principle employed by conventional neural networks (Gunn et al., 1997). SVM is a supervised learning method that is trained to discriminate samples from different classes. It belongs to a family of generalized linear classifiers that simultaneously minimize the empirical classification error and maximize the geometric margin. Hence, it is also known as a maximum margin classifier. There are many algorithms and softwares for SVM developed and available for download. LibSVM (Chang and Lin, 2001), a library for SVM, was used in this study. The radial basis function (RBF) kernel $\left(K(x, y)=e^{-r\|x-y\|^{2}}, \gamma>0\right)$ was used for all of the SVM models reported in this article.

Simply speaking, given a training set of protein sequences $\left(\mathbf{x}_{\mathrm{i}}, \mathrm{y}_{\mathrm{i}}\right), \mathrm{i}=1, \ldots, \mathrm{n}(\mathrm{n}$ is the number of sequences in the training set), where $\mathbf{x}_{\mathrm{i}} \in \mathrm{R}^{\mathrm{m}}$ ( $\mathrm{m}$ is 771 here) and $\mathrm{y}_{i} \in\{1,-1\}$ (1 means a protein is from the positive family, while -1 means it is a negative), the SVM requires the solution of the following problem:

$$
\begin{array}{ll} 
& \min _{w, b, \xi} \frac{1}{2} w^{T} w+C \sum_{i=1}^{n} \xi_{i} \\
\text { subject to } \quad & y_{i},\left(w^{T} \phi\left(\mathbf{x}_{i}\right)+b\right) \geq 1-\xi_{i} \\
& \xi_{i}, \geq 0
\end{array}
$$

Here, the training vectors, $\mathbf{x}_{\mathbf{i}}$, are mapped into a higher dimensional space by the function $\phi$. Then, SVM finds a linear hyper-plane with the maximum margin in this higher dimensional space to separate proteins of the positive family from the negatives. $\mathrm{C}$ is a parameter to penalize the model error.

There are two parameters in the SVM model while using RBF kernel, the penalty parameter $\mathrm{C}$ and the kernel parameter $\gamma$. Different sets of parameters $\mathrm{C}$ and $\gamma$ give different SVM models with different performances. Therefore, in order to construct a good SVM model that can accurately predict unknown proteins, the parameters $\mathrm{C}$ and $\gamma$ have to be determined before model construction. The method used here is the simple grid searching using twofold cross-validation. Basically, the training data set was evenly and randomly divided into two portions at first. Then each portion was used to construct a set of SVM models with different parameter pairs of $\mathrm{C}$ and $\gamma$ to predict the proteins in another portion. Overall prediction Matthew's correlation coefficients (MCCs) were calculated and compared. Finally, the parameter pair of C and $\gamma$ that gave the highest MCC was selected to train the model with the entire training set. Usually, overall accuracy is used to select the parameters, but MCC is better than accuracy to measure the performance of a model, especially when the data set is very biased to one of the two classes. The data sets in this study are all biased to the negatives. Therefore, MCC rather than accuracy was used to select the parameters $\mathrm{C}$ and $\gamma$. The process of selecting a good pair of parameters $\mathrm{C}$ and $\gamma$ for a training set is automatically conducted using a MatLab code.

\subsection{Model performance measurement}

To assess the performance of a classification model, the model needs to be tested on sequences not used in training the model. For multi-class SVM recognition models, overall recognition accuracy calculated from the recognitions was used to measure the performance. For two-class SVM classification models, however, different statistical parameters can be used to measure the goodness of the model from the prediction results for different purposes. Six different statistical parameters were calculated for measuring the performance of a model. The prediction result of a two-class SVM classification model is defined as:

Total Sequences (TS)

Predicted Positives (PP)

Predicted Negatives (PN)
Actual Positives (AP)

True Positives (TP)

False Negatives (FN)
Actual Negatives (AN)

False Positives (FP)

True Negatives (TN) 
Accuracy: Accuracy is the degree of conformity of a measured or calculated quantity to its actual (true) value. It is defined as the proportion of true positives and true negatives in the total sequences.

$$
\text { Accuracy }=(T P+T N) / T S
$$

Accuracy is used to measure overall performance of a model, but it does not take into account disparities in the numbers of positives and negatives. Hence, accuracy measurements obtained on testing sets with different distributions of positives and negatives are not directly comparable for measuring predictive power of the models. Therefore, there is a risk of losing the information because of the dominance of positives or negatives in the testing set.

Sensitivity: Sensitivity is a statistical measure of how well a binary classification model correctly identifies the positives. It is defined as the proportion of true positives to actual positive proteins in the testing set.

$$
\text { Sensitivity }=T P / A P
$$

Sensitivity is used to measure how sensitive is a model to the positives.

Specificity: Specificity is a statistical measure of how well a binary classification model correctly classifies proteins not belonging to a protein family. It is defined as the proportion of true negatives to actual negative proteins in the testing set.

$$
\text { Specificity }=T N / A N
$$

Specificity is used to measure how specific is a model to the positive protein family.

Positive predictive rate (PPR): PPR is the proportion of proteins with positive prediction results that are correctly predicted as the positive protein family. It reflects the probability that a positive prediction correctly reflects the underlying protein family. Here, PPR is defined as

$$
P P R=T P /(T P+F P)
$$

PPR is used to measure how precise are the positive predictions from a model.

Negative predictive rate (NPR): NPR is the proportion of proteins with negative prediction results that are correctly predicted as not belonging to the positive protein family. It reflects the probability that a negative prediction correctly rules out the underlying protein family. Here, NPR is defined as

$$
N P R=T N /(T N+F N)
$$

NPR is used to measure how precise are the negative predictions from a model.

Matthew's correlation coefficient (MCC): As accuracy does not take into account disparities in the numbers of positives and negatives, it cannot be used to measure the absolutely predictive power of a model and cannot be used to directly compare models tested on different datasets. MCC remedies this problem by integrating all information from the prediction results (Baldi et al., 2000). It is defined as

$$
M C C=\frac{T P \times T N-F P \times F N}{\sqrt{(T P+F N)(T P+F P)(T N+F N)(T N+F P)}}
$$

$\mathrm{MCC}$ is a prediction performance measurement that accounts for both over- and under-predictions. Its value ranges from -1 to 1 , where 0 represents a random prediction result. A positive value of MCC means a model predicts better than a random prediction, and a negative value indicates worse than a random prediction.

\subsection{Prediction of protein families}

To demonstrate the capability of the protein features for predicting whether an unknown protein belongs to a specific protein family or not based solely on its sequence, two-class SVM models were constructed from proteins in the training sets to predict the proteins in the testing sets. Two types of experiments were conducted to assess the performance of SVM models generated using the protein features developed in this study.

In the first type of experiment, the 4046 unique proteins of the 20 protein families were randomly divided into two even portions (proteins of each family were randomly divided into two even portions as training and testing, all the training portions from the 20 families were combined to serve as the training set and all 
the testing portions from the 20 families were combined to serve as the testing set). Then, the 2028 training proteins were used to construct two-class SVM models to predict the 2018 testing proteins. First, the training proteins of one family were assigned as the positives and the training proteins of the remaining 19 families were assigned as the negatives to train a two-class SVM classification model. Then, the SVM model was used to predict the 2018 testing proteins. To check whether the result was obtained just by chance due to the random division of the data set into the training and testing sets, twofold, fivefold, and 10 -fold cross-validations were conducted on the 2028 training proteins. The process was repeated 20 times by changing the positive protein family (each family is assigned to positive family once and only once) to generate 20 different two-class SVM classifiers for the 20 protein families.

In the first type of experiment, all 20 protein families were included in the training set to create the models for challenge with the 2018 testing proteins. In a real application, it is difficult to include all families in the training set as negatives. Therefore, a question arises: How good are the SVM models generated using the protein features when they are used to predict the proteins whose families were not contained in the training set? For this purpose, the second type of experiment was designed.

First, the 20 protein families were randomly divided into two groups, each group has 10 exclusive protein families. For group 1, the 2075 unique proteins were divided into two even portions as the training set (1040 proteins) and the testing set (1035 proteins). As in the first type of experiment, the training set was used to construct 10 two-class SVM classification models to predict whether an unknown protein belongs to a specific family or not. Then, these 10 two-class SVM models were used to predict the 1035 proteins in the testing set. At the same time, they were also used to predict the 1971 proteins in group 2 . The families of the 1971 proteins in group 2 were not included in the training set of group 1 . The same procedures were applied to group 2. The 1971 unique proteins were randomly divided into two even portions as the training set (988 proteins) and the testing set (983 proteins). The training set was then used to construct 10 two-class SVM classification models for predicting whether an unknown protein belongs to a specific family or not. Thereafter, the 10 SVM models were used to predict the 983 proteins in the testing set. Finally, they were used to predict the 2075 proteins in group 1. The families of the 2075 proteins in group 1 were not included in the training set of group 2. Twofold, fivefold, and 10-fold cross-validations were conducted for both cases to check whether the results were obtained just by chance because of randomly dividing the proteins into the training and testing sets.

\subsection{Recognition of protein families}

Two-class SVM classification models can predict whether a protein belongs to a specific protein family or not. For negative predictions, the family of the protein is not determined. The more general application is the recognition of protein families in which a specific protein family is not predicted to be (Yes or No), but a protein family has to be assigned clearly to an unknown protein. To demonstrate the ability of the protein features for recognizing protein families, multi-class SVM classification models were constructed using the training set and challenged with the testing set.

The data set of N (4046 for 20-class SVM mode; 2075 for 10-class SVM model of group 1; 1971 for 10-class SVM model of group 2) unique proteins were randomly divided into two even portions (by family). One portion (2028 proteins for 20-class SVM mode; 1040 protein for 10-class SVM model of group 1; 988 proteins for 10-class SVM model of group 2) was used as the training set to construct a multiclass SVM recognition model. Then, the other portion (2018 proteins for 20-class SVM mode; 1035 protein for 10-class SVM model of group 1; 983 proteins for 10-class SVM model of group 2) was used to challenge the multi-class SVM recognition model. As in the experiments of two-class SVM models, twofold, fivefold, and 10-fold cross-validations were conducted using the training set to check whether the results were obtained by chance due to dividing of the data set into the training set and testing set.

\subsection{Prediction and recognition confidence}

For a two-class SVM classification model, given a training set $\mathbf{x}_{\mathrm{i}} \in \mathrm{R}^{\mathrm{m}}$ ( $\mathrm{m}$ is 771 here) and $\mathrm{y}_{\mathrm{i}} \in\{1,-1\}, \mathrm{i}=1, \ldots, \mathrm{n}$ ( $\mathrm{n}$ is the number of proteins in the training set), the constructed SVM classification model computes a decision function $\mathrm{f}(\mathrm{x})$ such that the sign $(\mathrm{f}(\mathrm{x}))$ can be used to predict the label of any unknown protein $\mathrm{x}$. The prediction probability output from LibSVM (Lin et al., 2003) is an algorithmic improvement of Platt's probability (Platt, 2000) in which a posterior class probability is approximated by a sigmoid function 


$$
P(x)=\frac{1}{1+e^{A f(x)+B}}
$$

where the best parameters $\mathrm{A}$ and $\mathrm{B}$ are estimated by solving the following regularized maximum likelihood problem with a set of labeled samples $\left\{\left(\mathbf{x}_{i}, y_{i}\right)\right\}_{i=1}^{n}$ (with $\mathrm{N}_{+}$of the $\mathrm{y}_{\mathrm{i}}$ 's positive and $\mathrm{N}_{-}$of the $\mathrm{y}_{\mathrm{i}}$ 's negative).

$$
\begin{array}{r}
\min _{A, B}\left\{-\sum_{i=1}^{n}\left(t_{i} \log \left(p_{i}\right)+\left(1-t_{i}\right) \log \left(1-p_{i}\right)\right)\right\} \\
t_{i}= \begin{cases}\frac{N_{+}+1}{N_{+}+2} & \text { when } y_{i}=+1 \\
\frac{1}{N_{-}+2} & \text { when } y_{i}=-1\end{cases}
\end{array}
$$

As can be seen in the formula, the probabilities to assign the unknown protein $\mathrm{x}$ to positive family $+1\left(\mathrm{P}^{+}\right)$ or to negative family $-1\left(\mathrm{P}^{-}\right)$are complementary to 1 each other; that is, $\mathrm{P}^{+}+\mathrm{P}^{-}=1$. Therefore, the probabilities of a prediction can be used to assess the confidence of the prediction. When $\mathrm{P}^{+}=\mathrm{P}^{-}=0.5$, the SVM model predicts the unknown protein to both families with equal probability, and therefore no confidence at all. The difference of probabilities between $\mathrm{P}^{+}$and $\mathrm{P}^{-}$is the measure of prediction confidence with a value ranging from 0 to 1 . The bigger the value is, the more confident the prediction is.

There are many ways to extend a two-class classification approach to a multi-class classification method for calculation of prediction probability. Multi-class SVM in LibSVM adapts the most commonly used "one-against-one" strategy (Huang et al., 2006; Wu et al., 2004c), which is a special case of ECOC (error correcting output codes) (Allwein et al., 2001). At first, pairwise probabilities $r_{i j}$ (family $\mathrm{i}$ against $\mathrm{j}$, $\mathrm{i}, \mathrm{j}=1, \ldots, \mathrm{n}, \mathrm{n}$ is the number of families) can be estimated using the algorithm for calculating the twoclass probabilities:

$$
r_{i j}=\frac{1}{1+e^{A_{i j} f_{i j}+B_{i j}}}
$$

where $f_{i j}$ are the decision values from the two-class SVM classification model of family $i$ against $j$, and parameters $A_{i j}$ and $B_{i j}$ can be estimated using the same method for the two-class case. The probabilities $p_{i}$ to assign a protein to family $\mathrm{i}(\mathrm{i}=1, \ldots, \mathrm{n})$ were calculated from all the pairwise probabilities $\mathrm{r}_{\mathrm{ij}}$ by solving the following optimization problem:

$$
\begin{array}{r}
\min _{\mathrm{p}} \frac{1}{2} \sum_{i=1}^{n} \sum_{j(j \neq i)}\left(r_{i j} p_{i}-r_{i j} p_{i}\right)^{2} \\
\text { subject to } \quad \sum_{i=1}^{n} p_{i}=1 \text { and } p_{i} \geq 0
\end{array}
$$

The maximum probability $\mathrm{p}_{\mathrm{i}}$ is the recognition result for an unknown protein to be assigned to protein family $i$ with certainty of $\mathrm{p}_{\mathrm{i}}$. The probability of assigning a protein to a family in a multi-class SVM recognition model is different from that in a two-class SVM classification model. It was used to assess the confidence of the recognition directly.

\subsection{Sequence alignment}

Sequence alignment was conducted for all unique sequences in each of the 20 protein families. The Needleman-Wunsch algorithm (Needleman and Wunsch, 1970) was used to construct an optimal global alignment for each of all pairs of sequences in a protein family. The scoring matrix BLOSUM250 (Henikoff and Henikoff, 1992) was used in searching for the optimal global alignment, with a penalty value of 3 for opening a gap.

\section{RESULTS AND DISCUSSION}

To test our method, 20 protein families (Table 2) were selected randomly from the iProClass database of PIR (http://pir.georgetown.edu/) and filtered such that the number of protein sequences in each family was 
greater than 80 . Redundant sequences were removed before model construction and testing. There were 4383 protein sequences in the 20 families, including 337 redundant sequences (Table 2). After removal of the 337 redundant sequences, the remaining 4046 unique protein sequences were used to test our method. The unique protein sequences in each family were further randomly divided into two portions. One was used as a training set (2028 sequences) and the other as a testing set (2018 sequences; Table 2).

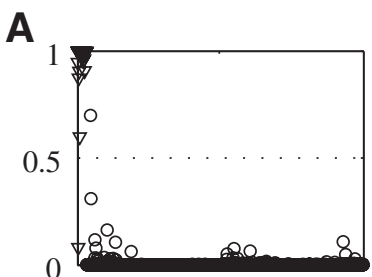

Pirsf000048

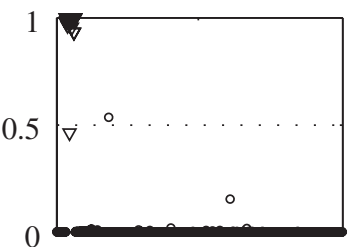

Pirsf000071

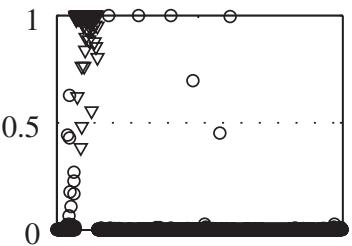

Pirsf000078

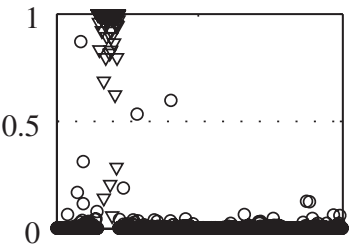

Pirsf000090

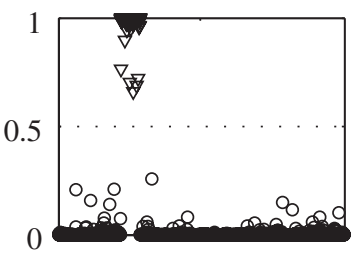

Pirsf000099

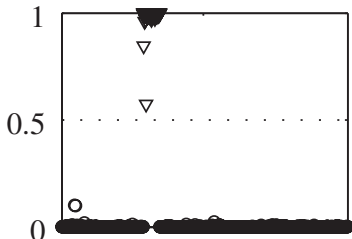

Pirsf000363

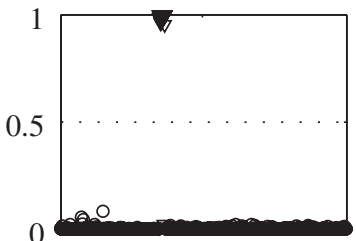

Pirsf000806

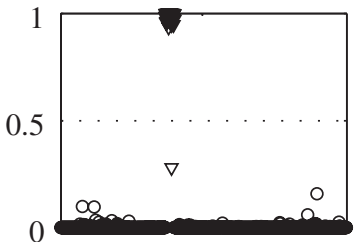

Pirsf001336

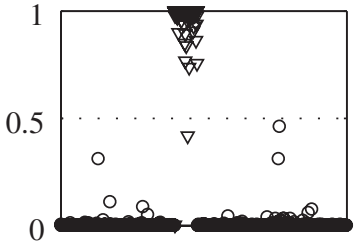

Pirsf001401

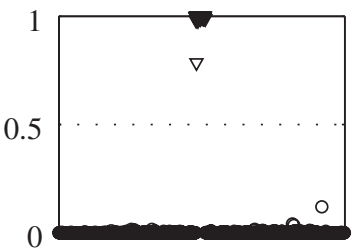

Pirsf001423

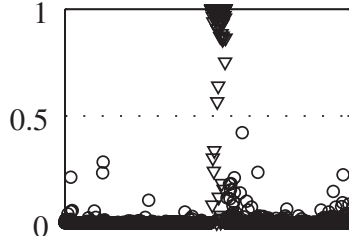

Pirsf000050

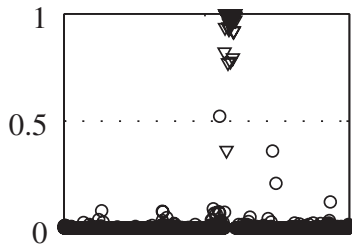

Pirsf000055

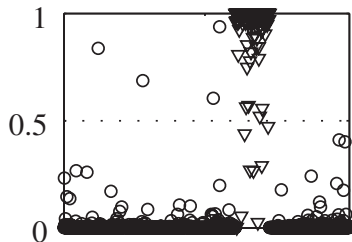

Pirsf000059

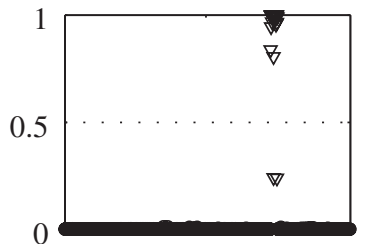

Pirsf000076

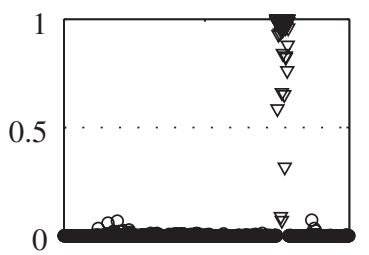

Pirsf000098

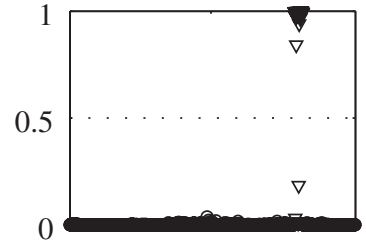

Pirsf000379

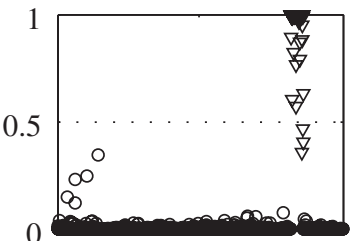

Pirsf000443

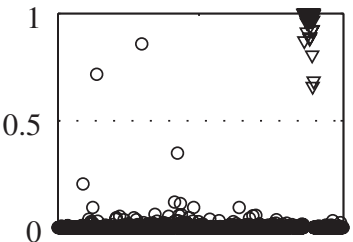

Pirsf001124

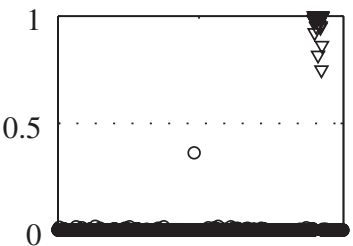

Pirsf001237

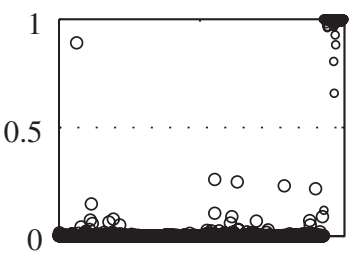

Pirsf002451

FIG. 1. Prediction results of two-class support vector machine (SVM) classification models. The $x$-axis represents the proteins in the testing set, and the $y$-axis shows the probabilities of assigning proteins to the positive family. The dash lines at the probability of 0.5 divide the predictions into negative predictions (bottom part) and positive predictions (top part). Actual (true) positive proteins were drawn in triangles and actual negative proteins in circles. The circles with probability of greater than 0.5 (false positives) and the triangles with probability of less than 0.5 (false negatives) are the incorrect predictions. The Protein Information Resources (PIR) identifications of positive protein families of the two-class SVM classification models are given along the $x$-axis. (A) Prediction results of the 20 two-class SVM classification models were constructed from all 20 protein families by using each family as positives and the other 19 families as negatives. (B) Prediction results of the 10 two-class SVM classification models were constructed from the 10 protein families in group 1 (the left two columns) and the 10 two-class SVM classification models constructed from the 10 protein families in group 2 (the right two columns) by taking each family as positives and the other nine families as negatives. 
B

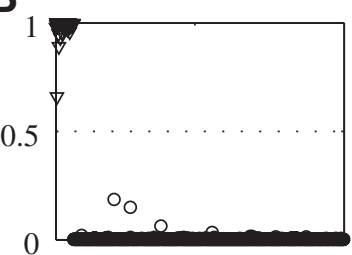

Pirsf000048

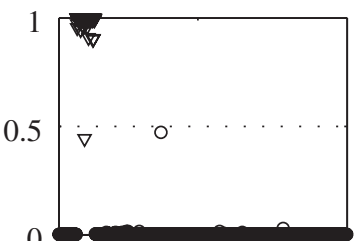

Pirsf000071

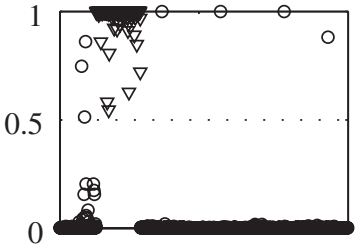

Pirsf000078

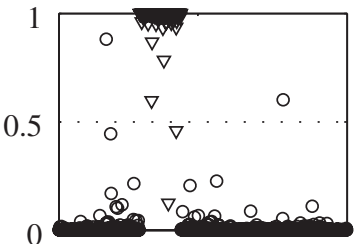

Pirsf000090

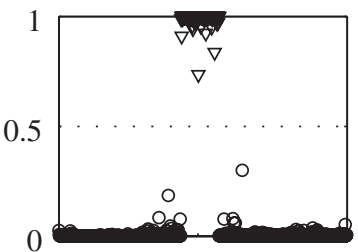

Pirsf000099

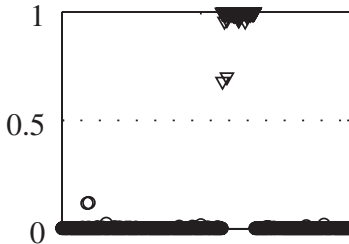

Pirsf000363

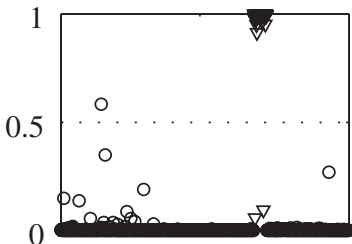

Pirsf000806

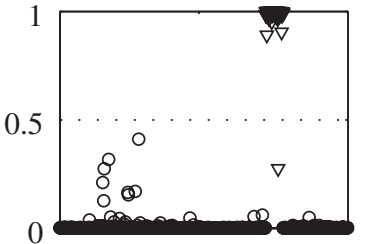

Pirsf001336

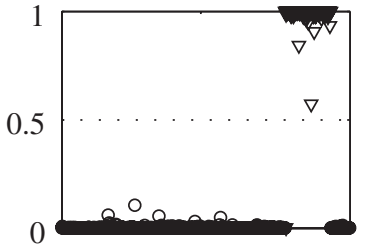

Pirsf001401

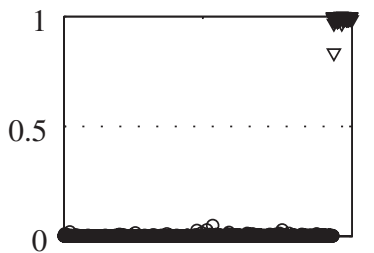

Pirsf001423

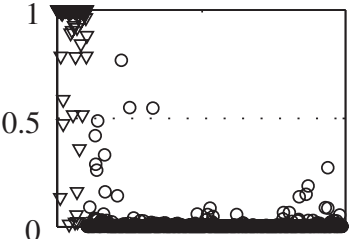

Pirsf000050

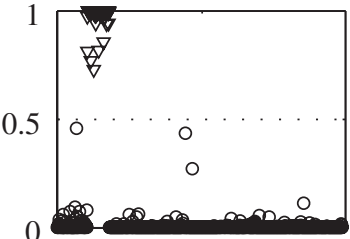

Pirsf000055

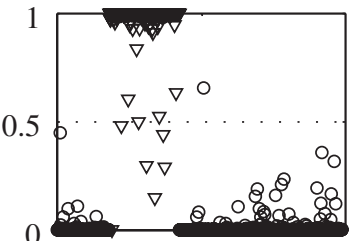

Pirsf000059

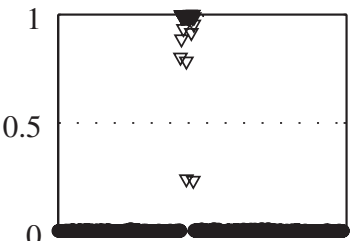

Pirsf000076

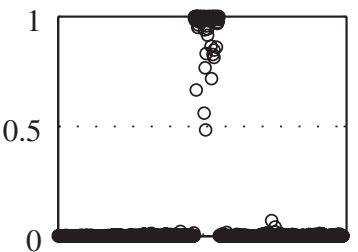

Pirsf000098

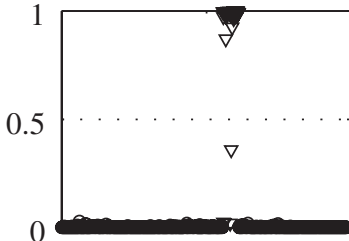

Pirsf000379

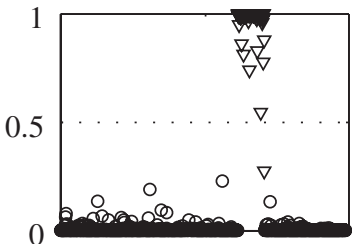

Pirsf000443

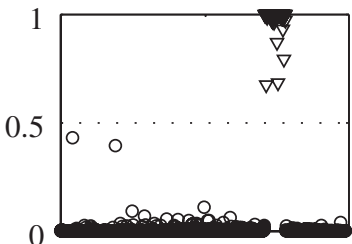

Pirsf001124

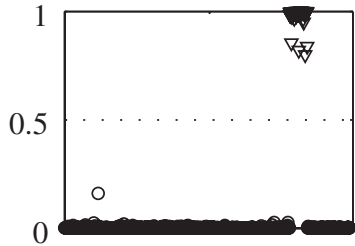

Pirsf001237

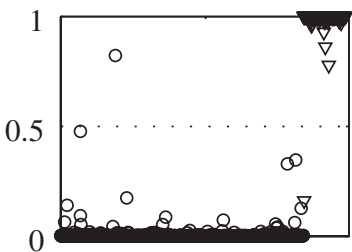

Pirsf002451

FIG. 1. (Continued).

Three types of experiments were designed to assess the ability of protein features, in conjunction with SVM, to predict and recognize protein family.

\subsection{Protein family prediction}

In the first type of experiment, the members of a single protein family were chosen from the training set (Table 2) and designated as the positives (called positive family thereafter). The sequences of the remaining 19 families in the training set were designated as the negatives (called negative family thereafter). A twoclass SVM classification model was then constructed for predicting the positive family. The sequences representing the 20 families in the testing set were used to challenge the two-class SVM model. The entire process was repeated by changing the positive family until each of the 20 protein families was treated as positive once and only once. Therefore, 20 predictions were obtained for each of the proteins in the testing set from the 20 different two-class SVM classification models for each of the 20 protein families (see materials and methods section for detail). The prediction results of the testing sequences from the 20 
two-class SVM models are plotted in Figure 1A. For all of the two-class SVM classification models (Figure 1A), very few of the proteins were incorrectly predicted (false negatives: triangles with a probability less than 0.5 ; false positives: circles with a probability greater than 0.5 ). Furthermore, the separation of positives from negatives was very clear with large prediction probability differences.

The performance of the 20 two-class SVM models was evaluated using six statistical parameters: accuracy, sensitivity, specificity, negative predictive rate, positive predictive rate, and Matthew's correlation coefficient (Table 3). Each of the six statistical parameters demonstrated that the 20 two-class SVM classification models can accurately predict the protein families of unknown proteins based on their primary sequences alone. Eighteen of the two-class SVM classification models fit the training sets at $100 \%$, and the remaining two models at $99.95 \%$ (Table 4). The average prediction accuracy of the 20 two-class SVM classification models reached 0.9984 with a small standard deviation value of 0.0018 .

Since accuracy does not take into account disparities in the numbers of positives and negatives, it cannot be used to measure the absolutely predictive power of a model. It also cannot be used to directly compare models that are developed for different datasets. Matthew's correlation coefficient (MCC) remedies this problem by integrating information from all of the prediction results and was also used as plotted in black bars in Figure 2. The average MCC reached 0.9663 with a standard deviation value of 0.0294 , indicating that the two-class SVM classification models constructed using the protein features can be used to accurately predict protein family and are also stable for the different protein families.

To evaluate whether the results were obtained just by chance from randomly dividing the sequences of each families into the training and testing sets, twofold, fivefold, and 10-fold cross-validation analyses were conducted for the 20 two-class SVM models using only the training set. The cross-validation results listed in Table 4 for the two-class SVM classification models using the 20 protein families had prediction accuracies of better than 0.98 , which is very similar to the results based on the original testing dataset (0.99). Therefore, there is a low probability that the prediction results observed by randomly dividing the protein sequences into the training set and the testing set were obtained by chance alone.

The SVM classification model can predict the class of a protein, but also can calculate the probability of a protein being assigned to different families. The probabilities can be used to estimate the confidence of the prediction (see Section 2 for computing the probability and prediction confidence). The average

Table 3. Summary of Prediction Results of Two-Class SVM Classification Models

\begin{tabular}{|c|c|c|c|c|c|c|}
\hline Models & Accuracy & Sensitivity & Specificity & $P P R$ & $N P R$ & $M C C$ \\
\hline \multicolumn{7}{|l|}{ Maximum } \\
\hline Group 1 & 1.0000 & 1.0000 & 1.0000 & 1.0000 & 1.0000 & 1.0000 \\
\hline Group 2 & 1.0000 & 1.0000 & 1.0000 & 1.0000 & 1.0000 & 1.0000 \\
\hline All & 1.0000 & 1.0000 & 1.0000 & 1.0000 & 1.0000 & 1.0000 \\
\hline \multicolumn{7}{|l|}{ Minimum } \\
\hline Group 1 & 0.9932 & 0.9565 & 0.9920 & 0.9578 & 0.9977 & 0.9323 \\
\hline Group 2 & 0.9888 & 0.9223 & 0.9966 & 0.9694 & 0.9906 & 0.8825 \\
\hline All & 0.9941 & 0.8835 & 0.9968 & 0.9632 & 0.9938 & 0.8780 \\
\hline \multicolumn{7}{|l|}{ Mean } \\
\hline Group 1 & 0.9984 & 0.9806 & 0.9989 & 0.9922 & 0.9993 & 0.9812 \\
\hline Group 2 & 0.9971 & 0.9750 & 0.9994 & 0.9958 & 0.9972 & 0.9678 \\
\hline All & 0.9984 & 0.9754 & 0.9995 & 0.9925 & 0.9988 & 0.9663 \\
\hline \multicolumn{7}{|l|}{ SD } \\
\hline Group 1 & 0.0022 & 0.0137 & 0.0025 & 0.0143 & 0.0009 & 0.0237 \\
\hline Group 2 & 0.0038 & 0.0295 & 0.0011 & 0.0096 & 0.0036 & 0.0389 \\
\hline All & 0.0018 & 0.0286 & 0.0009 & 0.0110 & 0.0016 & 0.0294 \\
\hline \multicolumn{7}{|l|}{$\mathrm{CV}$} \\
\hline Group 1 & 0.0022 & 0.0155 & 0.0025 & 0.0148 & 0.0009 & 0.0242 \\
\hline Group 2 & 0.0038 & 0.0301 & 0.0011 & 0.0097 & 0.0036 & 0.0396 \\
\hline All & 0.0018 & 0.0293 & 0.0009 & 0.0110 & 0.0016 & 0.0295 \\
\hline
\end{tabular}

PPR, negative predictive rate; NPR, positive predictive rate; MCC, Matthews correlation coefficient; SD, standard deviation; $\mathrm{CV}$, coefficient variance; Group 1, SVM generated from proteins of 10 families in group 1; Group 2, SVM generated from proteins of 10 families in group 2; All, SVM generated from proteins of all 20 families. 
Table 4. Accuracy of Two-Class SVM Models

\begin{tabular}{|c|c|c|c|c|c|c|}
\hline Positive family & Positives & Negatives & Fitting & $10-f-C V$ & $5-f-C V$ & $2-f-C V$ \\
\hline \multicolumn{7}{|c|}{ Models of all protein families } \\
\hline sf000048 & 64 & 1964 & 1 & 1 & 0.9995 & 0.9985 \\
\hline sf000071 & 68 & 1960 & 1 & 1 & 1 & 0.9985 \\
\hline sf000078 & 160 & 1868 & 1 & 0.9956 & 0.9965 & 0.9946 \\
\hline sf000090 & 149 & 1879 & 0.9995 & 0.9975 & 0.9970 & 0.9965 \\
\hline sf000099 & 138 & 1890 & 0.9995 & 0.9980 & 0.9975 & 0.9970 \\
\hline sf000363 & 119 & 1909 & 1 & 1 & 1 & 1 \\
\hline sf000806 & 47 & 1981 & 1 & 0.9956 & 0.9970 & 0.9961 \\
\hline sf001336 & 63 & 1965 & 1 & 0.9995 & 0.9995 & 1 \\
\hline sf001401 & 164 & 1864 & 1 & 0.9975 & 0.9965 & 0.9965 \\
\hline sf001423 & 68 & 1960 & 1 & 1 & 1 & 0.9990 \\
\hline sf000050 & 104 & 1924 & 1 & 0.9951 & 0.9936 & 0.9896 \\
\hline sf000055 & 78 & 1950 & 1 & 0.9985 & 0.9975 & 0.9946 \\
\hline sf000059 & 242 & 1786 & 1 & 0.9911 & 0.9911 & 0.9911 \\
\hline sf000076 & 47 & 1981 & 1 & 1 & 1 & 1 \\
\hline sf000098 & 87 & 1941 & 1 & 1 & 1 & 0.9995 \\
\hline sf000379 & 57 & 1971 & 1 & 0.9995 & 0.9995 & 0.9995 \\
\hline sf000443 & 93 & 1935 & 1 & 0.9965 & 0.9961 & 0.9941 \\
\hline sf001124 & 73 & 1955 & 1 & 0.9936 & 0.9931 & 0.9906 \\
\hline sf001237 & 61 & 1967 & 1 & 1 & 1 & 0.9990 \\
\hline sf002451 & 146 & 1882 & 1 & 0.9995 & 0.9995 & 0.9961 \\
\hline \multicolumn{7}{|c|}{ Models of protein families in group 1} \\
\hline sf000048 & 64 & 976 & 1 & 1 & 1 & 1 \\
\hline sf000071 & 68 & 972 & 1 & 1 & 1 & 1 \\
\hline sf000078 & 160 & 880 & 1 & 0.9952 & 0.9942 & 0.9923 \\
\hline sf000090 & 149 & 891 & 0.9990 & 0.9962 & 0.9981 & 0.9913 \\
\hline sf000099 & 138 & 902 & 1 & 0.9971 & 0.9971 & 0.9952 \\
\hline sf000363 & 119 & 921 & 1 & 1 & 1 & 1 \\
\hline sf000806 & 47 & 993 & 1 & 0.9981 & 0.9971 & 0.9962 \\
\hline sf001336 & 63 & 977 & 1 & 1 & 1 & 0.9990 \\
\hline sf001401 & 164 & 876 & 1 & 0.9981 & 0.9990 & 0.9990 \\
\hline sf001423 & 68 & 972 & 1 & 1 & 1 & 1 \\
\hline \multicolumn{7}{|c|}{ Models of protein families in group 2} \\
\hline sf000050 & 104 & 894 & 1 & 0.9939 & 0.9919 & 0.9899 \\
\hline sf000055 & 78 & 920 & 1 & 0.9949 & 0.9960 & 0.9929 \\
\hline sf000059 & 242 & 756 & 1 & 0.9868 & 0.9868 & 0.9818 \\
\hline sf000076 & 47 & 951 & 1 & 1 & 1 & 1 \\
\hline sf000098 & 87 & 911 & 1 & 1 & 1 & 1 \\
\hline sf000379 & 57 & 941 & 1 & 0.9990 & 0.9990 & 0.9990 \\
\hline sf000443 & 93 & 905 & 1 & 0.9970 & 0.9970 & 0.9949 \\
\hline sf001124 & 73 & 925 & 1 & 0.9959 & 0.9919 & 0.9919 \\
\hline sf001237 & 61 & 937 & 1 & 1 & 1 & 1 \\
\hline sf002451 & 146 & 852 & 1 & 0.9980 & 0.9980 & 0.9980 \\
\hline
\end{tabular}

prediction confidence for the correct predictions (0.9945) was higher than that of the incorrect predictions (0.9190) for all of the two-class SVM models. Therefore, protein features examined provide reliable, efficient classification and prediction of protein family assignment.

These SVM analyses showed that protein families can be accurately predicted from primary amino acid sequences by using the protein features. One caveat is that all negative protein families were included in the training set. In real-life applications for protein classification, it is difficult to include all protein families in the training set as negatives. The question then arises as to how accurate the models would be in correctly predicting that a protein does not belong in a family when the proteins from the negative families are not included in the training set. 


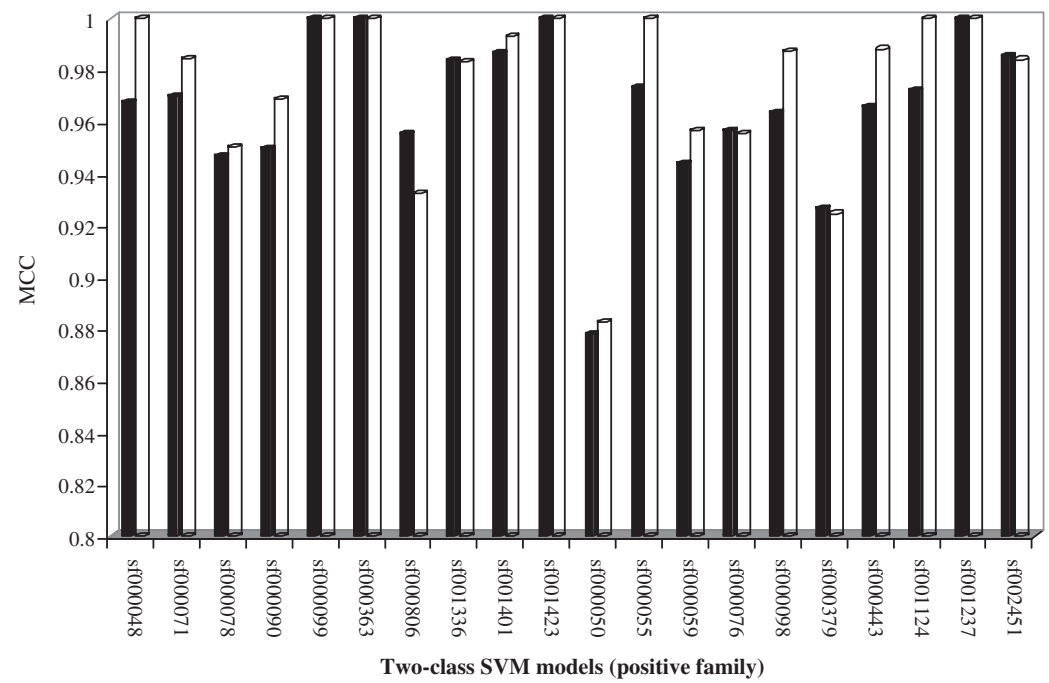

FIG. 2. Bar chart of performances using Matthew's correlation coefficient (MCC) for the two-class support vector machine (SVM) classification models. The models are depicted by the Protein Information Resources (PIR) identifications of the positive protein families in the SVM models listed in Table 2. The MCCs were calculated from the predictions of testing sets as described in the text. MCCs for the predictions based on the 2018 testing proteins using the 20 two-class SVM classification models (constructed from all 20 protein families by taking proteins in each family as positives and the proteins in other 19 families as negatives) are in black bars. MCCs for predictions based on the 1035 testing proteins using the 10 two-class SVM classification models constructed from the 10 protein families in group 1 (by taking proteins in each family as positives and the proteins in other 9 families as negatives) are in white bars. MCCs for predictions based on the 983 testing proteins using the 10 two-class SVM classification models constructed from the 10 protein families in group 2 (by taking proteins in each family as positives and the proteins in other 9 families as negatives) are in white bars.

Since it would be useful to understand the false positive rate for misclassifying proteins when the family for that particular protein is not present in the training set, the second type of experiment was designed and conducted (see Section 2 for details). The 20 protein families were randomly divided into two groups of 10 mutually exclusive protein families (Table 2). Within each group, two-class SVM models were developed using the sequences of one family at a time from the training set as the positives. The sequences from the remaining nine families were used as the negatives. The sequences of testing set within each group were used to challenge the SVM classification models developed and the prediction results are shown in Figure 1B. Very few of the proteins were incorrectly predicted (false negatives are shown as triangles with a probability less than 0.5 ; false positives are shown as: circles with a probability greater than 0.5 ) by the two-class SVM classification models. The separation of positives from negatives was very clear with large prediction probability difference.

The statistical performances of the SVM models calculated from the prediction results are given in Table 3.

Based on six statistical parameters, the two-class SVM classification models built only from group 1 or 2 family members performed similar to the models built from all 20 protein families. The average prediction accuracies were 0.9984 with a standard deviation of 0.0022 for group 1, and 0.9971 with a standard deviation of 0.0038 for group 2. The MCCs calculated from the prediction results from the testing sequences were plotted in white bars for models from group 1 and in white bars for models from group 2 in Figure 2. The average MCC of models from group 1 was 0.9811 with a standard deviation of 0.0237 . The average MCC of models from group 2 was 0.9678 with a standard deviation of 0.0389 .

To determine whether the results obtained from randomly dividing the sequences of each of the families into the training and testing sets were obtained just by chance, twofold, fivefold, and 10-fold cross validations were conducted for the two-class SVM models using only the training set. The cross-validation results are listed in Table 4 for all two-class SVM classification models from both groups 1 and 2 . Prediction accuracies were better than 0.98 . The findings are very similar to the results from predictions on the initial testing dataset ( 0.998 for group 1 and 0.997 for group 2). Based upon these results, there is a low 
probability that the prediction results obtained by randomly dividing the protein sequences into the training and testing sets were due to chance alone. The average prediction confidences of correct predictions ( 0.9927 and 0.9885 for groups 1 and 2, respectively) were higher than the average prediction confidences of incorrect predictions ( 0.8307 and 0.8978 for group 1 and 2, respectively).

To assess the false positive rates ( 1 - specificity) for proteins in cases where proteins from a specific protein family are not present in the training sets, the 10 two-class SVM classification models from group 1 were used to predict the proteins of group 2 (both training and testing). The results are shown in Figure 3A (average false positive rate: 0.0088 ). In the same manner, the 10 two-class SVM classification models constructed from group 2 were applied to predict protein families from the sequences in group 1 and the results are shown in Figure 3B (average false positive rate: 0.0022). Most of the two-class SVM classification models gave false positive rates of less than 0.01. The highest false positive rate, 0.0655 , was obtained from the SVM model for prediction of membrane dipeptidase proteins (pirsf001124). An average false positive rate of 0.0055 was obtained for the 20 two-class SVM classification models to predict proteins when sequences from their families were not included in the training sets used to construct the models. This was slightly higher than the average false positive rate obtained when sequences from the
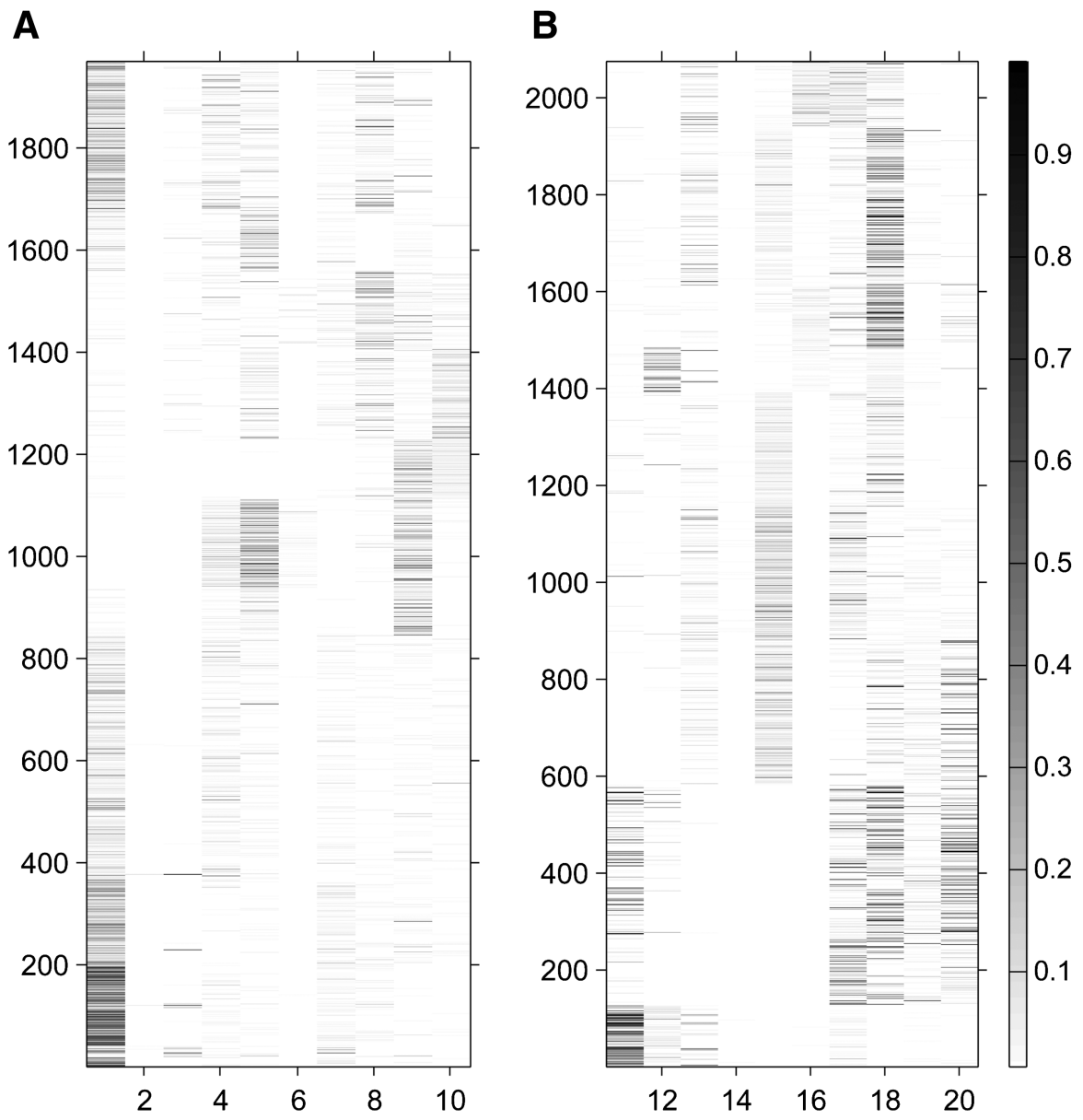

FIG. 3. Heat map of prediction results of two-class support vector machine (SVM) classification models on proteins whose families were not included in the training models. The $x$-axis shows the SVM models that are identified by the identification (ID) numbers (Table 2) of the positive protein family used in a particular SVM model. The $y$-axis shows the testing proteins, sorted by their protein family IDs as listed in Table 2. The prediction probabilities for the positives in the SVM models are grey coded according to the grey-bar shown on the right side. Incorrect predictions (false positives) are the black or darker lines with probabilities greater than 0.5 . White or lighter predictions are correct predictions. (A) Prediction results of the 1971 proteins in group 2 using the 10 two-class SVM classification models constructed from the 10 protein families in group 1. (B) Prediction results of the 2075 proteins in group 2 using the 10 two-class SVM classification models constructed from the 10 protein families in group 1. 
negative protein families were included in model construction ( 0.0009 for models from group one and two, and 0.0005 for models from all 20 families; Table 4), but still quite low. Thus, the low false positive rates for these models allow their use on large datasets that frequently contain many more negatives than positives.

\subsection{Protein family recognition}

A two-class SVM classification model can be used to predict whether an unknown protein belongs to a family based solely on its sequence. One drawback is that an unknown protein may be predicted to belong to more than one or none of the 20 families. Therefore, a recognition model is needed to uniquely assign a protein to a single protein family. To investigate whether the protein features reported here can be used to accurately recognize protein families solely from the amino acid sequences, a third type of experiment was designed and conducted (see Section 2 for details). In this analysis, a multi-class SVM recognition model was constructed from the training set and the model was used to recognize the protein families in the testing set. The 2028 protein sequences from the training set (Table 2) of all 20 families were used to generate a 20-class SVM recognition model and the 2018 proteins in the testing set of the 20 families were used to challenge the model. All 2028 proteins were correctly classified into their families in the training set by the recognition models. The correct protein families were predicted for 1995 of the 2018 test proteins (Figure $4 \mathrm{~A})$. The 20-class SVM recognition model showed a very high overall recognition accuracy of 0.9886 and also gave a much higher recognition confidence for the correct recognitions than for the incorrect recog-
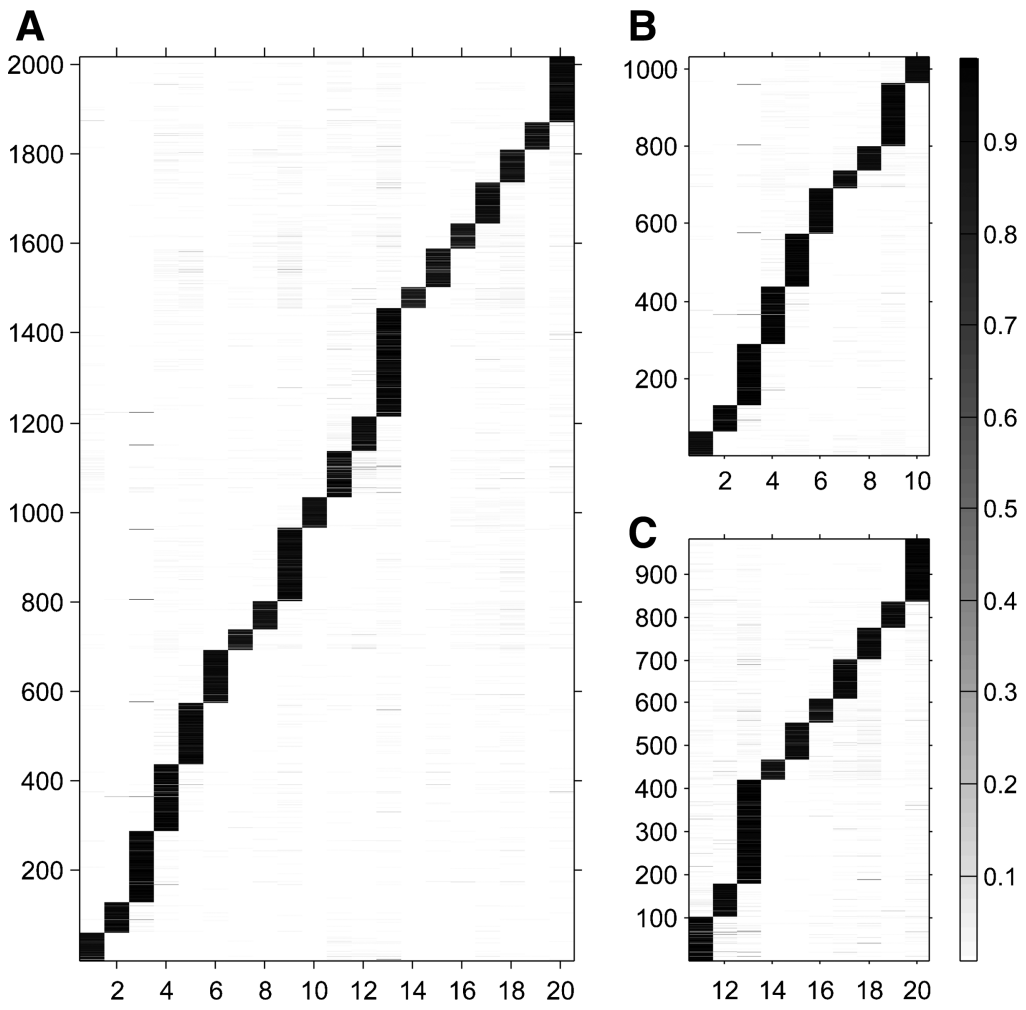

FIG. 4. Heat map of recognition results of the multi-class support vector machine (SVM) recognition models. Columns represent protein families identified by the protein family identifications (IDs; Table 2) on the $x$-axis, and rows represent proteins in the testing sets sorted by the protein family IDs on the $y$-axis (number of proteins is different for different families; Table 2). The grey map depicts the probabilities of proteins being recognized as the corresponding families by the multi-class SVM recognition models. (A) The recognition results of the 2018 testing proteins by the $20-$ class SVM recognition model constructed from the training set containing all 20 protein families. (B) The recognition results of the 1035 testing proteins by the 10-class SVM recognition model constructed from the training set of 10 protein families in group 1. (C) The recognition results of the 983 testing proteins by the 10-class SVM recognition model constructed from the training set of 10 protein families in group 2. 


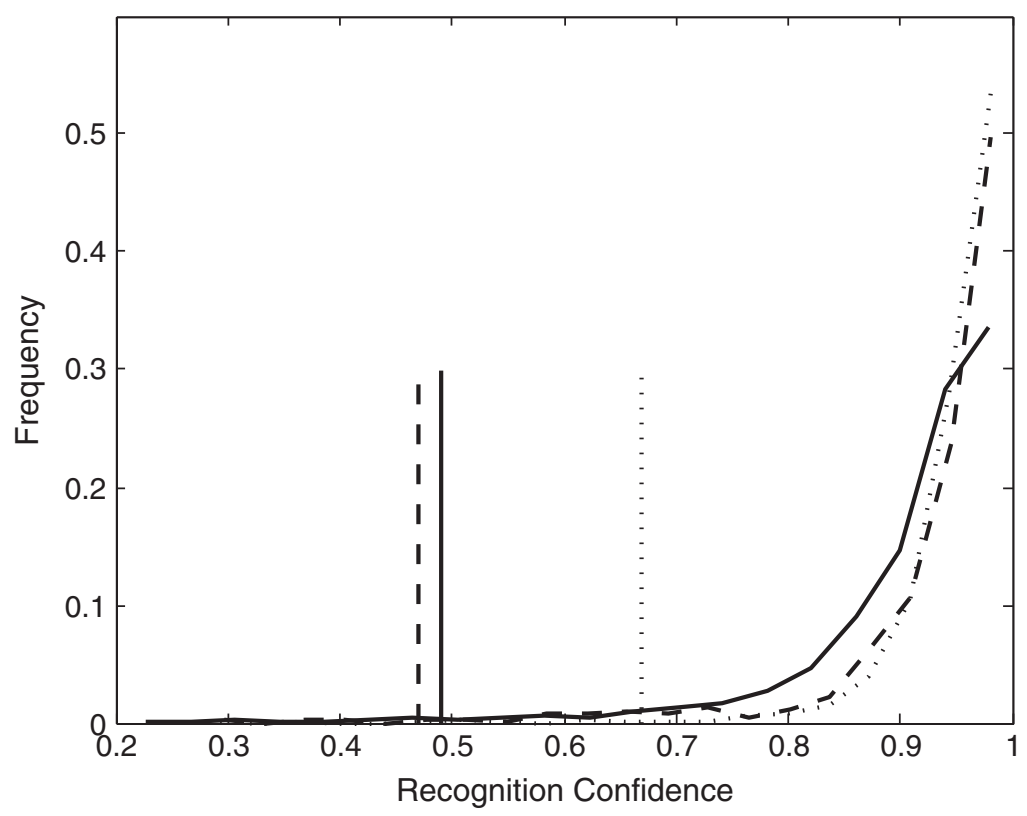

FIG. 5. Frequency distribution of recognition results of the multi-class support vector machine (SVM) recognition models versus recognition confidence. The recognition confidence distribution of the 1995 correct recognitions from the 20-class SVM recognition model is the solid line. The recognition confidence distribution of the 1028 correct recognitions from the 10-class SVM recognition model of group 1 is the dotted line. The recognition confidence distribution of the 975 correct recognitions from the 10-class SVM recognition model of group 2 is the dashed line. As a comparison, the average recognition confidences for the incorrect recognitions ( 23 for the 20-class model; seven for the 10-class model in group 1; eight for the 10-class model in group 2) are depicted as vertical lines using the same line types as for the models.

nitions. The distribution of the recognition confidences of the 1995 proteins correctly recognized in the testing set by the SVM recognition model is plotted as a solid black line in Figure 5. The average recognition confidence for the 1995 correct recognitions was 0.9075 , compared to a low average recognition confidence of 0.4891 for the 23 incorrect recognitions (the vertical black line in Figure 5).

To assess whether the high recognition accuracy was obtained just by chance from randomly dividing the sequences of each of the families into the training and testing sets, twofold, fivefold, and 10-fold crossvalidations using the 2028 training proteins from the 20 families were conducted. High overall recognition accuracies (Table 5) were observed, indicating the recognition models were very stable to changes in the size of the training sets and the manner in which the proteins were divided between the training and testing sets.

The same experimental procedures were then applied to the group 1 and 2 protein families and similar results were obtained. The recognitions of 1035 testing proteins in group 1 from the 10-class SVM recognition model trained by using the 1040 training proteins in group 1 are plotted in Figure 4B. The recognitions of 983 testing proteins in group 2 from the 10-class SVM recognition model trained by using

Table 5. Accuracy of Multi-Class SVM Models

\begin{tabular}{lcccccc}
\hline Model & Families & Proteins & Fitting & $2-f-C V$ & $5-f-C V$ & $10-f-C V$ \\
\hline All & 20 & 2028 & 1 & 0.9896 & 0.9946 & 0.9946 \\
Group 1 & 10 & 1040 & 1 & 0.9971 & 0.9981 & 0.9971 \\
Group 2 & 10 & 988 & 1 & 0.9828 & 0.9939 & 0.9939 \\
\hline
\end{tabular}

All, SVM generated from proteins of all 20 families; Group 1, SVM generated from proteins of 10 families in group 1; Group 2, SVM generated from proteins of 10 families in group 2; 2-f-CV, twofold cross-validation; 5-f-CV, fivefold cross-validation; 10-f-CV: 10 -fold cross-validation. 


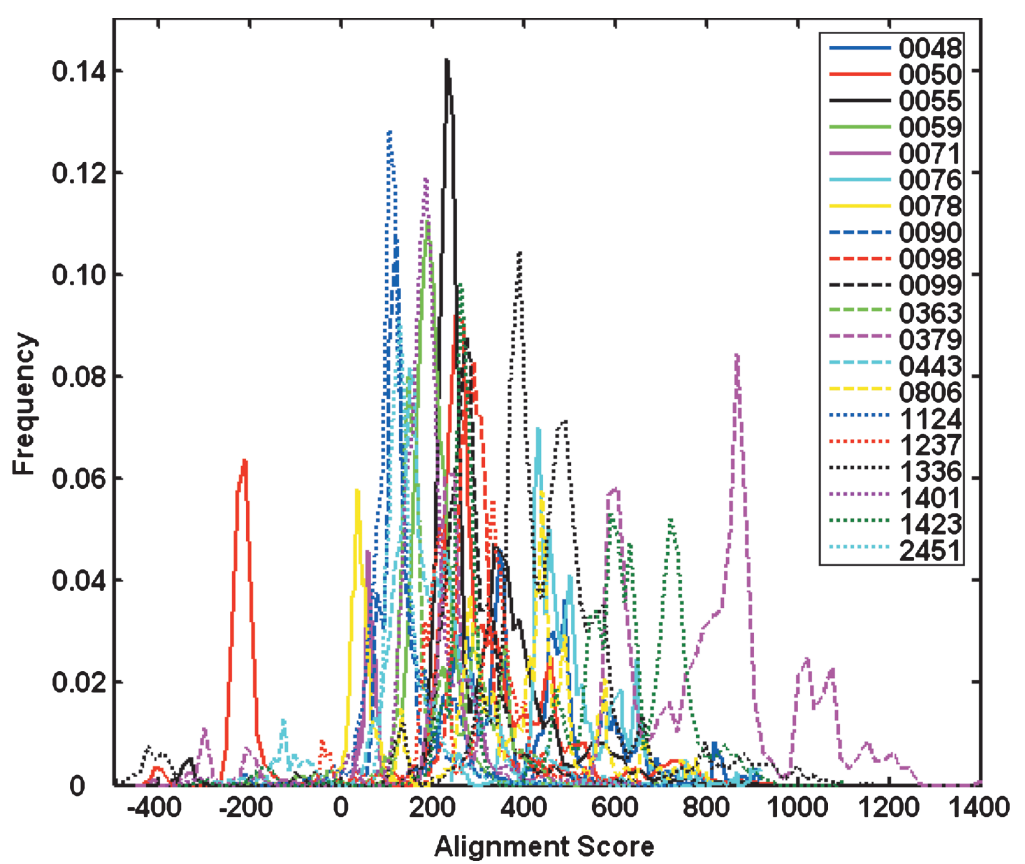

FIG. 6. Frequency distribution of global alignment scores between proteins in each of the 20 protein families. The labels for the 20 protein families are the last four characters of the protein family identifications (IDs) listed in Table 2.

the 988 training proteins in group 2 are plotted in Figure 4C. High recognition accuracies of 0.9932 and 0.9919 were obtained for group 1 and group 2, respectively. The distribution of the recognition confidences of the 1028 testing proteins in group 1 that were correctly recognized by the SVM recognition model is plotted as a dotted line in Figure 5. The distribution of the recognition confidences of the 975 testing proteins in group 2 that were correctly recognized by the SVM recognition model is plotted as a dashed line in Figure 5. The average recognition confidence for the 1028 correct recognitions in group 1 is 0.9502 , compared to a much lower average recognition confidence of 0.6887 for the 7 incorrect recognitions (the vertical dotted line in Fig. 5). The average recognition confidence for the 975 correct recognitions in group 2 is 0.9318 , compared to a much lower average recognition confidence of 0.4702 for the eight incorrect recognitions (the vertical dashed line in Fig. 5). Comparing these results with the results from the 20-class SVM recognition model, it can be seen that the number of protein families and number of proteins do not affect the accuracy of the recognition models.

The twofold, fivefold, and 10-fold cross-validations using the 1040 training proteins from the 10 families in group 1 and the 988 training proteins from the 10 families in group 2 were conducted, respectively, and high overall recognition accuracies (Table 5) were observed. This further confirms that the recognition models are very stable to changes in the size of the training sets and the manner in which the proteins are divided between the training and testing sets.

\subsection{Homology between proteins}

To check whether the performances of protein family prediction and recognition models are due to the method reported in this paper or due to homology differences between proteins in the 20 protein families, global alignments between sequences were conducted for each of the 20 protein families. The scores from sequence alignments were used to measure the homology or similarity between proteins. The frequency distributions of scores for each protein family were calculated based on 100 even bins (from minimum score to maximum score) and plotted in Figure 6. It is clear that the 20 protein families cover a large range of alignment scores. Therefore, the proteins used to test our method are diverse regarding homology or similarity between proteins in each family, especially for protein family PIRSF000050. Furthermore, the scores distributions overlap much for different protein families. The analysis of homology between proteins 
on the 20 protein families used to test our method demonstrated that it is unlikely that the performances of the models are due to very different homologies of the protein families used in this study.

\section{CONCLUSION}

The three types of experiments for protein family prediction and recognition demonstrated that the protein features assessed in this article are suitable for accurately and quickly predicting and recognizing protein family based solely on protein sequence. The SVM models used all available protein features. Variable selection was not needed. The lengths of the 4046 unique protein sequences were in the range of 45-902 amino acids. The protein features reported work well on proteins with large differences in sequence lengths and should aid the analysis of the ever-expanding protein sequences databases.

\section{DISCLOSURE STATEMENT}

No competing financial interests exist.

\section{REFERENCES}

Allwein, E.L., Schapire, R.E., and Singer, Y. 2001. Reducing multiclass to binary: a unifying approach for margin classifiers. J. Mach. Learn. Res. 1, 113-141.

Anfinsen, B.C. 1973. Principles that govern the folding of protein chains. Science 181, 223-230.

Baldi, P., Brunak, S., Chauvin, Y., et al. 2000. Assessing the accuracy of prediction algorithms for classification: an overview. Bioinformatics 16, 412-424.

Baldi. P., Chauvin, Y., Hunkapiller, T., et al. 1994. Hidden Markov models of biological primary sequence information. Proc. Natl. Acad. Sci. USA 91, 1059-1063.

Chang, C.-C., and Lin, C.-J. 2001. LIBSVM: a library for support vector machines [Software]. Available at: www.csie.ntu.edu.tw/ cjlin/libsvm. Accessed June 15, 2009.

Cortes, C., and Vapnik, V. 1995. Support-vector networks. Mach. Learn. 20, 273-297.

Gunn, S.R., Brown, M., and Bossley, K.M. 1997. Network performance assessment for neurofuzzy data modeling, $313-$ 324. In: Liu, X., Cohen, P. and Berthold, M. eds. Advances in Intelligent Data Analysis. Reasoning about Data. Springer-Verlag, New York.

Henikoff, S., and Henikoff, J.G. 1992. Amino acid substitution matrices from protein blocks. Proc. Natl. Acad. Sci. USA 89, 10915-10919.

Huang, T.-K., Weng, R.C., and Lin, C.-J. 2006. Generalized Bradley-Terry models and multi-class probability estimates. J. Mach. Learn. Res. 7, 85-115.

Karchin, R., Karplus, K., and Haussler, D. 2002. Classifying g-protein coupled receptors with support vector machines. Bioinformatics 18, 147-159.

Keerthi, S.S., and Lin, C.J. 2003. Asymptotic behaviours of support vector machines with gaussian kernel. Neural Comput. 15, 1667-1689.

Krogh, A., Brown, M., Mian, I., et al. 1994. Hidden Markov models in computational biology. J. Mol. Biol. 235, 15011531.

Lapinsh, M., Gutcaits, A., Prusis, P., et al. 2002. Classification of G-protein coupled receptors by alignment independent extraction of principal chemical properties of primary amino acid sequences. Protein Sci. 11, 795-805.

Lin, H.-T., Lin, C.-J., and Weng, R.C. 2003. A note on Platt's probabilistic outputs for support vector machines. Available at: www.csie.ntu.edu.tw/ cjlin/papers.html. Accessed June 15, 2009.

Needle, S.B., and Wunsch, C.D. 1970. A general method applicable to the search for similarities in the amino acid sequence of two proteins. J. Mol. Biol. 48, 443-453.

Platt, J.C. 2000. Probabilities for SV machine, 61-74. In: Smola, A., Bartlett, P., Scholkopf, B., et al., eds. Advances in Large Margin Classifiers. The MIT Press, Cambridge, MA.

Sgourakis, N.G., Bagos, P.G., Papasaikas, P.K., et al. 2005. A method for the prediction of GPCRs coupling specificity to G-proteins using refined profile hidden Markov models. BMC Bioinform. 6, 104-115.

Vries, J.K., Munshi, R., Tobi, D., et al. 2004. A sequence alignment-independent method for protein classification. Appl. Bioinform. 3, 137-148. 
Wu, C.H., Huang, H., Nikolskaya, A., et al. 2004a. The iProClass integrated database for protein functional analysis. Comput. Biol. Chem. 28, 87-96.

Wu, C.H., Nikolskaya, A., Huang, H., et al. 2004b. PIRSF: family classification system at the Protein Information Resource. Nucleic Acids Res. 32, D112-D114.

Wu, T.-F., Lin, C.-J., and Weng, R.C. 2004c. Probability estimates for multi-class classification by pairwise coupling. J. Mach. Learn. Res. 5,975-1005.

Address correspondence to: Dr. Huixiao Hong Division of Systems Toxicology National Center for Toxicological Research

U.S. Food and Drug Administration 3900 NCTR Road Jefferson, AR 72079

E-mail: Huixiao.Hong@fda.hhs.gov 